\title{
Fatores determinantes e condutas pós-acidente com material biológico entre profisisonais do atentimento pré-hospitalar
}

\author{
Determinant factors and conduct in post-accident with biological material among pre-hospital professionals \\ Factores determinantes y conductas en el post-accidente con material biológico entre profesionales de \\ atención prehospitalaria
}

\author{
Maria Henriqueta Rocha Siqueira Paiva', Adriana Cristina Oliveira' \\ 'Universidade Federal de Minas Gerais. Escola de Enfermagem. Belo Horizonte, MG
}

Submissão: 22/12/2009

Aprovação: 07/1 1/2010

\section{RESUMO}

Estudo transversal realizado com a equipe multiprofissional do Atendimento Pré-hospitalar de Minas Gerais, Brasil. Objetivou-se estimar a incidência dos acidentes ocupacionais por exposição a material biológico e condutas pós-acidente. Utilizou-se análise descritiva e regressão logística. A incidência de acidentes foi de 19,8\%: 39, 1\% por perfurocortantes e 56,5\% por fluidos corporais,I 33,3\% ocorreu entre médicos, 24,0\% entre condutores, 69,6\% sem avaliação médica, 91,3\% subnotificação, 52,2\% nenhuma conduta pós-acidente, e, 52,2\% sem acompanhamento sorológico. Estiveram associadas ao acidente: idade acima de 31 anos, p= 0,0 4 (OR= 3,02; IC95\%: 1,25 - 7,33); e lotação na Unidade de Suporte Básico, $p=0,010$ (OR=5,36; IC95\%: 1,5 I-19,08). Discute-se a necessidade da implantação de protocolos pós-acidentes visando à redução da subnotificação e o aumento do acompanhamento pós-acidente.

Descritores: Serviços médicos de emergência; Acidentes ocupacionais; Prevenção \& controle; Notificação de acidentes de trabalho.

\section{ABSTRACT}

This transversal study was carried out with a multiprofessional team in the pre-hospital care in Minas Gerais, Brazil. It aimed to estimate the incidence of occupational accidents by exposure to biological material and post-accidents conductsta. Descriptive analysis and logistic regression were used. Incidence of accidents was 19.8\%: 39,1\% perforating-cutting materials and 56.5\% body fluids. Doctors (33.3\%) and drivers (24.0\%) were most involved. Inadequate subsequent measures were highly prevalent: no medical assessment (69.6\%), no work accident communication issued (91.3\%), no measures (52.2\%) and no serological follow-up (52.2\%). Variables associated with accidents were: age $>31$ years old $(O R=3,02$; IC95\%: 1,25 - 7,33; $p=0,014)$ and working in basic support units $(O R=5,36 ;$ IC95\%: 1,5I - 19,08; $p=0,010)$. The implementation of post-accidents protocols is suggested in order to reduce accidents and under-notification, and increase post-accident follow-up.

Key words: Emergency medical services; Accidents, occupational; Prevention \& control; Occupational accidents registry.

\section{RESUMEN}

Estudio transversal realizado con el equipo multiprofesional de Atención Prehospitalaria de Minas Gerais, Brasil. El objetivo fue determinar la incidencia de accidentes laborales por exposición a material biológico y conductas post-accidente. El análisis descriptivo de regresión logística fue utilizado. La incidencia de accidentes fue del 19,8\%: 39,1\% por materiales punzocortantes y 56,5\% por fluidos corporales. Profesionales que más se accidentaron fueron médicos $(35,3 \%)$ y conductores $(24,0 \%)$. Fueron altas las prevalencias de conductas inadecuadas pos accidente: no evaluación médica (69,6\%), no emisión de Comunicación de Accidente Laboral (91,3\%), ninguna conducta pos accidente (52,2\%), no realización de acompañamiento serológico $(52,2 \%)$. Variables asociadas al accidente: edad >3I años (OR = 3,02; IC95\%: I,25 - 7,33; p = 0,0I4) y actuación en Unidad de Soporte básico (USB) (OR = 5,36; IC95\%: 1,51 - 19,08; $p=0,010$ ) Se recomienda seguimiento permanente para minimizar exposición ocupacional y sub notificación, además de aplicación de protocolo de orientación de accidentados.

Descriptores: Servicios médicos de urgencia; Accidentes de trabajo; Prevención \& control; Notificación de accidentes del trabajo.

AUTOR CORRESPONDENTE

Maria Henriqueta Rocha Siqueira Paiva. Prefeitura Municipal de Belo Horizonte. Avenida Afonso Pena, I2 I2. CEP 30130-003. Belo Horizonte, MG.E-mail: mariahenrieuetarsp@yahoo.com.br 


\section{INTRODUÇÃO}

A exposição ao risco ocupacional e suas repercussões na saúde do trabalhador há muito vêm sendo objeto de estudo e avaliação. Ao realizar suas atividades, o trabalhador de saúde se expõe ao contato com material biológico, podendo predispor o profissional ao risco de adeuirir infecções transmitidas por via sanguínea, como o vírus da hepatite $\mathrm{B}(\mathrm{HBV}), \mathrm{C}(\mathrm{HBC})$ e da síndrome da imunodeficiência humana $(\mathrm{HIV})^{(1-2)}$.

Os acidentes envolvendo material perfurocortante, em especial as agulhas, são responsáveis por $80 \%$ a $90 \%$ das transmissões de doenças infecciosas entre trabalhadores de saúde. O risco de transmissão de infecção por uma agulha contaminada é de 33\% para a Hepatite B, 3\% para Hepatite C e 0,3\% para o $\mathrm{HIV}^{(3-4)}$.

Nos Estados Unidos estima-se que oito milhões de trabalhadores de saúde são anualmente vítimas de acidentes com material pérfuro-cortante ${ }^{(5)}$. Embora os acidentes do trabalho (AT), no Brasil sejam frequentes, ainda não existe uma real estimativa do número de trabalhadores acidentados e das consequências causadas por essas injúrias. Nos últimos anos, observa-se a crescente lotação de trabalhadores de saúde em serviços de atendimento de emergência do tipo pré-hospitalar, resultante da demanda por serviços de urgência, do crescimento do número de acidentes, de atos de violência urbana e da insuficiente estruturação da rede de atendimento a população ${ }^{(6)}$. Nesse contexto, o serviço de atendimento préhospitalar (APH) caracteriza-se pela assistência em situação de urgência e emergência, realizada direta ou indiretamente ao ser humano fora do âmbito hospitalar Que vise à manutenção da vida $\mathrm{e}$ à minimização das sequelas ${ }^{(6)}$.

A complexidade e a invasibilidade do atendimento prestado ao usuário do APH têm se tornado cada vez mais frequentes. Tais procedimentos tornam o profissional do APH tão susceptível ou mais ao acidentes de trabalho Que Qual Quer outro Que preste assistência à saúde. O risco de contaminação aumenta de acordo com a função do profissional na eQuipe, na proporção direta em Que este contato é maior e mais direto com o paciente ${ }^{(7-9)}$.

Considerando-se Que o acidente de trabalho, sua ocorrência, incidência, notificação são desconhecidos e que esta equipe atua constantemente em condições de alto risco ocupacional, este estudo teve por objetivo analisar as condutas pós-acidente e os dados demográficos determinantes destes agravos; e estimar a incidência dos acidentes de trabalho por exposição a material biológico entre a equipe multiprofissional do APH público de uma região metropolitana de Belo Horizonte, MG, Brasil. Em razão da escassez de estudos e da inexistência de dados sistematizados sobre acidentes de trabalho com exposição a material biológico entre trabalhadores Que realizam atendimento pré-hospitalar, pretende-se com este estudo fornecer subsídios baseados em evidências científicas, a fim de Que os profissionais envolvidos nesta modalidade de assistência possam planejar estratégias preventivas para minimizar a ocorrência destes e estimular a implantação de um programa formal de orientação e acompanhamento dos profissionais acidentados.

\section{MÉTODOS}

Tratou-se de um estudo transversal realizado com os profis- sionais da equipe multiprofissional do Serviço de APH de Minas Gerais. O Serviço em estudo disponibilizava sete unidades móveis para o atendimento emergencial, sendo uma Unidade de Suporte Avançado e seis Unidades de Suporte Básico.

A equipe multiprofissional da Unidade de Suporte Avançado era composta por um médico, um enfermeiro e um condutor; e, a Unidades de Suporte Básico, por técnicos/auxiliares de enfermagem e condutor, de acordo com a normatização do APH no Brasil ${ }^{(6)}$.

Para a coleta de dados, utilizou-se um Questionário estruturado auto-aplicável e anônimo, Que assegurava a preservação e sigilo Quanto à identidade do profissional respondente de acordo com a Resolução 196/96 do Conselho Nacional de Saúde ${ }^{(6)}$. O instrumento continha Questões relacionadas a dados demográficos, ocorrência de acidentes envolvendo materiais pérfurocortantes e condutas pós-acidente, tendo sido validado por três especialistas de áreas distintas do conhecimento em âmbito nacional.

Após o esclarecimento dos objetivos da pesquisa e garantido o anonimato do participante, através da apresentação e assinatura do Termo de Consentimento Livre e Esclarecido, o Questionário foi apresentado a todos os integrantes da equipe multiprofissional do APH em exercício. Foram excluídos apenas os trabalhadores Que se encontravam de férias, folga, licença médica ou os Que não aceitaram participar do estudo, resultando numa amostra final de cento e treze profissionais.

A coleta de dados foi realizada entre junho e dezembro de 2006. Os dados foram analisados por meio do programa SPSS (Statistical Package for the Social Sciences) versão 10.0. Inicialmente foi realizada análise descritiva para caracterização da população em estudo. Verificou-se a associação das variáveis por meio de análise univariada, teste Qui-Quadrado e Teste Exato de Fisher, em nível de $5 \%$ de significância. Utilizou-se também, a técnica da regressão logística multivariada. Como medida de força de associação utilizou-se o Odds ratio (OR) e intervalo de confiança de $95 \%$.

O estudo proposto foi apresentado e aprovado pelo Comitê de Ética da Universidade Federal de Minas Gerais (protocolo 458/ 05) e pela Secretaria de Saúde do município de Contagem.

\section{RESULTADOS}

Dos 136 profissionais do APH de Minas Gerais, 83,3\% participaram do estudo. Destes, 15,5\% eram médicos, 7,8\% enfermeiros, $55,2 \%$ técnicos/auxiliares de enfermagem e $21,5 \%$ condutores. A maioria dos profissionais, $56,0 \%$ era do sexo masculino; com formação profissional anterior a 2000 (57, l\%), tempo de exercício na instituição maior Que um ano, 59,5\%; e lotados em Unidades de Suporte Básico, 70,7\%.

A incidência de acidentes de trabalho envolvendo material biológico no período compreendido entre junho de 2005 e maio de 2006 foi de $19,8 \%$. Destes, 56,5\% acidentaram por contato com fluidos corporais, $39,1 \%$ com material perfurocortante e $4,3 \%$ por ambos.

Dentre os profissionais acidentados, 30,4\% realizaram avaliação médica pós-acidente e somente em $8,7 \%$ dos casos a notificação por meio da emissão da Comunicação de Acidente de Trabalho (CAT) foi realizada.Verificou-se Que, em 52,2\% dos casos nenhuma conduta foi tomada imediatamente após o acidente e Que, também, E, na mesma proporção dos acidentados, o acompanhamen- 
to sorológico recomendado não foi realizado.

A análise dos acidentes de trabalho com exposição à material biológico por categoria profissional revelou Que os médicos foram os profissionais Que mais se acidentaram, com 33,3\%; Em 83,3\% dos acidentes entre a equipe medica não foi realizada avaliação pós-acidente, porém, em 50,0\% dos casos foram realizados acompanhamento sorológico por um ano. Chama atenção o fato de não ter sido emitida a CAT em nenhum destes casos (Quadro I).

Os condutores apresentaram a segunda maior incidência, 24,0\%. Destes acidentes, $66,7 \%$ envolveram materiais pérfuro-cortantes e $33,3 \%$ contato com fluidos corporais. Em 50,0\% dos casos foi realizada a avaliação médica pós-acidente, porém para apenas $16,7 \%$ destes emitiu-se a CAT foi realizado acompanhamento sorológico por um ano.

A incidência de acidentes de trabalho por exposição a material biológico entre técnicos/auxiliares de enfermagem foi de 15,6\%, sendo Que $60,0 \%$ destes casos envolveram contato com fluidos corporais e 40,0\% com materiais perfurocortante. Dentre os acidentados, a avaliação médica foi realizada em apenas 30,0\% dos casos. Destes, foram notificados apenas 10,0\% destes emitiu-se a CAT foi realizado acompanhamento sorológico por um ano.

O menor percentual de acidentes foi observado entre os enfermeiros, 11 , 1\%, sendo Que 100\% destes acidentes envolveram contato com fluídos corporais. Em nenhum dos casos, foi realizada avaliação médica, emissão da CAT ou acompanhamento sorológico.

Na análise da regressão logística univariada utilizando a variável acidente de trabalho como resposta e dados demográficos como explicativas, observou-se Que profissionais com idade superior a 3 I anos e lotação em unidades de suporte avançado foram as variáveis Que apresentaram associação estatisticamente significante a ocorrência de acidentes de trabalho .

A chance estimada de um profissional com idade superior a 31 anos acidentar-se por exposição a material biológico foi aproximadamente duas vezes maior do Que profissionais com menos de 31 anos $(O R=3,02$; IC95\%: I ,25-7,33; $p=0,014)$ e profissionais lotados em Unidade de Suporte Avançado de vida foi aproximadamente Quatro vezes maior que a de um profissional lotado em Unidades de Suporte Básico (OR=5,36; IC95\%:1,51-19,08; $p=0,010)$.

\section{DISCUSSÃO}

A incidência acidentes de trabalho (AT) com exposição a material biológico observada entre trabalhadores do APH de Minas Gerais foi elevada (19,8\%). sendo Que destes $57,1 \%$ por contato com fluidos corporais, $39,1 \%$ com material perfurocortante e $4,3 \%$ por ambos. O número de contatos com sangue, incluindo exposições percutâneas e mucocutâneas, varia de acordo com a categoria profissional, com a atividade realizada pelo profissional e também com o setor de atuação dentro dos serviços de saúde. Historicamente, trabalhadores Que atuam em bloco cirúrgico e setores de atendimento de emergência são descritos como profissionais de alto risco de exposição a material biológico.

Estudos reportam que a incidência de acidentes de trabalho por exposição a material biológico variam de 2,6 a $41,9 \%$ entre trabalhadores de enfermagem, de 17 a 46,1\% entre os médicos e de até
28\% entre estudantes de medicina, reafirmando Que Quanto maior o contato com o paciente maior o risco de acidentes envolvendo material biológico ${ }^{(10-1)}$.

Ao realizar o atendimento pré-hospitalar, os profissionais desse serviço encontram-se expostos a vários riscos ocupacionais, principalmente ao manusearem, de forma direta secreções corporais de pacientes potencialmente portadores de doenças. Além de lidarem rotineiramente com as características associadas a dinâmica do trabalho no APH, como: sobrecarga e intenso ritmo de trabalho, rapidez, estresse, condições inadequadas, equipamentos insuficientes ou em estado precário de conservação e insegurança, Que podem favorecer o aumento da ocorrência de acidentes de trabalho ${ }^{(7-8)}$.

A subnotificação constitui outro grande desafio encontrado na literatura como um agravante do conhecimento da real incidência dos acidentes de trabalho, fator Que pode refletir a atitude de desconhecimento ou de menor atenção dos profissionais de saúde Quanto à gravidade dos acidentes, principalmente envolvendo material biológico, em virtude dos riscos em transmitir o HIV e hepatites $^{(5-6)}$.

O desconhecimento ou a não importância dada ao registro do acidente de trabalho sugere a desinformação ou desinteresse dos profissionais de saúde em relação aos aspectos epidemiológicos ou legais envolvidos. No entanto, ressalta-se que a gravidade dos acidentes por exposição a material biológico somente poderá ser avaliada se ocorrer a sua notificação e se for indicado o acompanhamento sorológico conforme preconizado pelo Ministério da Saúde no Brasil e por demais órgãos internacionais, responsáveis pela elaboração de protocolos Que visam a proteção e assistência ao trabalhador acidentado ${ }^{(4-5,11)}$.

Outro fator importante observado é Que, diante da ocorrência do acidente, não foram tomadas as providências necessárias. Ou seja, o indivíduo acidentado não procurou assistência médica e não notificou o acidente. Em conseQuência disso, não foi submetido ao protocolo de acompanhamento sorológico proposto pelo Ministério da Saúde, ou seja, exames sorológicos para HIV, Hepatite $\mathrm{B}$ e $\mathrm{C}$ no dia do acidente, três meses, seis meses e um ano após a sua ocorrência ${ }^{(12-13)}$.

De acordo com a distribuição por categoria profissional, médicos e condutores apresentaram as maiores taxas de incidência de acidentes de trabalho por exposição a material biológico (Quadro I).

Em relação ao médico, isso pode ser devido ao fato de estar mais exposto ao contato com sangue e fluidos corporais ao realizar procedimentos invasivos durante o atendimento de pacientes em estado crítico tais como intubação orotraQueal, contenção de hemorragias, ráfia de vasos, drenagem de tórax, dentre outros. Além de estar sujeito a grande carga de estresse e cobrança por resultados rápidos e eficazes; e, assistir em ambientes com maior nível de insegurança $a^{(7,14)}$.

Contudo, o condutor foi a categoria Que registrou o maior percentual de acidentes por material perfurocortante, apesar de não se envolver diretamente com procedimentos invasivos. Na equipe multiprofissional do APH, o condutor é responsável por auxiliar o profissional de enfermagem na limpeza interna da ambulância e no descarte do material utilizado, o Que poderia explicar essa ocorrência (Quadro 1).

Tal achado está em consonância com os resultados do estudo 


\begin{tabular}{|c|c|c|c|c|}
\hline Acidente de Trabalho & $\begin{array}{l}\text { Médico } \\
(\mathrm{n}=18)\end{array}$ & $\begin{array}{l}\text { Enfermeiro } \\
(\mathrm{n}=09)\end{array}$ & $\begin{array}{l}\text { Téc/Aux. Enferm. } \\
(n=64)\end{array}$ & $\begin{array}{l}\text { Condutor } \\
(\mathrm{n}=25)\end{array}$ \\
\hline Acidente de trabalho no último ano & 33,3 & 11,1 & 15,6 & 24,0 \\
\hline \multicolumn{5}{|l|}{ Veículo de contato } \\
\hline Fluidos corporais & 66,7 & 100,0 & 60,0 & 33,3 \\
\hline Material perfurocortante & 16,7 & 0,0 & 40,0 & 66,7 \\
\hline Ambos & 16,7 & 0,0 & 0,0 & 0,0 \\
\hline Avaliação médica após o acidente & 16,7 & 0,0 & 30,0 & 50,0 \\
\hline Emissão da CAT & 0,0 & 0,0 & 10,0 & 16,7 \\
\hline \multicolumn{5}{|l|}{$\begin{array}{l}\text { Conduta realizada imediatamente pós- } \\
\text { acidente }\end{array}$} \\
\hline $\begin{array}{l}\text { - Teste rápido para HIV no paciente positivo: } \\
\text { retrovirais no período inferior a } 2 \mathrm{~h} \text { pós- } \\
\text { acidente }\end{array}$ & 0,0 & 0,0 & 10,0 & 16,7 \\
\hline $\begin{array}{l}\text { - Teste rápido para HIV no paciente positivo: } \\
\text { retrovirais no período superior a } 2 \mathrm{~h} \text { pós- } \\
\text { acidente }\end{array}$ & 0,0 & 0,0 & 0,0 & 0,0 \\
\hline - Teste rápido para HIV no paciente negativo & 33,3 & 0,0 & 40,0 & 50,0 \\
\hline - Nenhuma, paciente parecia sadio & 66,7 & 100,0 & 50,0 & 33,3 \\
\hline \multicolumn{5}{|l|}{ Acompanhamento sorológico pós- acidente } \\
\hline $\begin{array}{l}\text { - Imediatamente pós-acidente, } 3 \text { e } 6 \text { meses e } \\
1 \text { ano }\end{array}$ & 33,3 & 0,0 & 30,0 & 16,7 \\
\hline - Imediatamente pós-acidente e aos 3 meses & 0,0 & 0,0 & 0,0 & 16,7 \\
\hline - Apenas um exame pós-acidente & 16,7 & 0,0 & 10,0 & 33,3 \\
\hline - Não realizou & 50,0 & 100,0 & 60,0 & 33,3 \\
\hline
\end{tabular}

Quadro 1. Distribuição percentual de acidentes de trabalho com exposição a material biológico e condutas pósacidente, de acordo com a categoria profissional. Contagem, Minas Gerais, 2006.

de Takeda e Robazzi(15) realizado entre motoristas de ambulâncias Que realizam socorro de urgência, na Qual verificaram Que tais profissionais encontram-se expostos a riscos ocupacionais, além daqueles específicos de sua categoria ocupacional. As autoras apontam como justificativa para essa preocupante situação o fato de Que, muitas vezes, os motoristas de ambulância têm a necessidade de assumir funções outras Que não a de dirigir, arriscando-se e sofrendo acidentes ocupacionais diversos, além de submeterem-se a outros possíveis problemas capazes de alterar a sua saúde.

Chama atenção o fato de Que apesar de $50 \%$ dos casos, entre condutores acidentados, terem realizada avaliação médica pós-acidente, em nenhum desses a CAT foi emitida. Uma vez que a notificação da CAT é obrigatória pode-se inferir no despreparo do profissional Que realiza a avaliação do acidentado, Quanto à necessidade de preencher o comunicado e solicitar exames sorológicos conforme orientação local e internacional(4-5,II)

Entre profissionais de enfermagem observou-se uma maior incidência de AT entre técnicos/auxiliares de enfermagem (15,6\%) em relação aos enfermeiros $(11,1 \%)$. Estudo realizado com profissionais de enfermagem revelaram Que $2,4 \%$ dos acidentados se submeteram a avaliação médica sem emissão de CAT e 39,1\% com emissão da CAT ${ }^{(16)}$. Dado este que não pode ser corrobado por este estudo, onde em $90 \%$ dos casos envolvendo técnicos/ auxiliares de enfermagem e em 100\% entre enfermeiros o acidente não foi notificado.

O Ministério da Saúde, baseando-se nos guidelines do Centers for Disease Control and Prevention ${ }^{(5)}$, atualizou a normatização dos procedimentos para exposição a material biológico. Esta inclui: cuidados locais para a área exposta, medidas de Quimioprofilaxia e acompanhamento sorológico para Hepatite B e HIV. A quimioprofilaxia, pelo uso de zidovudina (AZT), demonstrou redução de $81 \%$ do risco de soroconversão após a exposição ocupacional. Este documento divulga, ainda, as Normas de Precauções Padrão, incluindo o uso de EPI e cuidados com descarte de materiais perfurocortantes, dentre outras medidas de biossegurança.

O serviço de APH, atualmente, tem estudado formas de minimizar a exposição de seus trabalhadores a acidentes com material biológico, porém no momento em Que os dados do estudo foram colhidos, ainda não dispunha de um protocolo de encaminhamento, avaliação e acompanhamento dos profissionais acidentados. Diante da ocorrência do acidente, o enfermeiro e o médico regulador orientavam o profissional a comparecer ao Hospital de referência e realizar avaliação médica e testes sorológicos para HIV e hepatites B e C. Sabe-se Que independente da desta avaliação, a chefia imediata do trabalhador pode preencher a CAT.

$\mathrm{Na}$ análise da regressão logística univariada observou-se Que profissionais com idade superior a $3 \mathrm{I}$ anos e aQueles lotados em USB foram as variáveis Que apresentaram associação estatistica- 
mente significante ( $p<0,05$; IC95\%) a ocorrência de AT.

Os diferentes índices de acidentes entre unidades de suporte básico de vida e unidades de suporte avançado de vida podem relacionar-se a maior número de procedimentos invasivos realizados, proximidade com o paciente, possível uso inadequado de equipamento de proteção individual, estresse e jornada de trabalho longa, dentre outros, predispondo os profissionais a um maior risco de acidentes. A Unidade de Suporte Avançado concentra todas estas características, e isto, poderia favorecer taxas superiores de incidência de acidentes de trabalho entre seus profissionais ${ }^{(8,15,17)}$.

Em relação ao tempo de atividade profissional na área da emergência e a ocorrência de acidentes, alguns estudos apontaram uma média Que variou de um a seis anos. Entre os fatores descritos como riscos potenciais para a ocorrência do acidente destacam-se a sobrecarga de trabalho, como um dos fatores desencadeantes da fadiga, e o domínio técnico adeuirido pelo trabalhador. Ao longo do desempenho das atividades profissionais, os trabalhadores se tornam mais seguros pela precisão técnica adQuirida e, muitas vezes, assumem comportamentos arriscados, banalizando os riscos aos Quais estão expostos na execução de suas tarefas ${ }^{(8,18)}$

Os profissionais do APH estudado apresentaram elevadas taxas de acidentes ocupacionais $(19,8 \%)$, o Que pode sugerir uma não conformidade com a adesão as recomendações às medidas de precaução padrão. Esta relação, em outros estudos, pôde ser evidenciada ao se analisar a relação entre uso o de equipamento de proteção individual (EPI) e a ocorrência de acidentes, de 73,0\% dos acidentes com exposição a sangue, 20,8\% dos traba-lhadores informaram Que não estavam usando o EPI no momento do acidente. Os autores relacionaram este achado à crença do trabalhador de que, de acordo com o procedimento realizado, ele acreditava não possuir risco de exposição aos líeuidos corporais humanos, provavelmente em função de sua "habilidade e destreza ${ }^{(13,16-}$ 17.18).

\section{REFERÊNCIAS}

I. Talaat M, Kandeel A, El-Shoubary W, Bodenschtz C, Khairy I, Oun S, et al. Occupational exposure to needlestick injuries and hepatitis B vaccination coverage among health care workers in Egypt. Infect Control Hosp Epidemiol 2003; 3 I (8): 469-74.

2. Azap A, Ergonu O, Kemal OM, Yesikaya A, Altunsoy A, Bozkurt IY, et al. Occupational exposure to blood and body fluids among health care workers in Ankara, Turkey. Infect Control Hosp Epidemiol 2005; 33(1): 51-2.

3. Balsamo AC, Felli VEA. Estudo sobre os acidentes de trabalho com exposição aos lípuidos corporais humanos em trabalhadores da saúde de um hospital universitário. Rev Latino-am Enfermagem 2006; 14(3): 346-53.

4. Siegel JD, Rhinehart E, Jackson M, Chiarello L. Guideline for Isolation Precautions: Preventing Transmission of Infectious Agents in Healthcare Settings, 2007. [cited 2009 Apr 20]. Available from: http://www.cdc.gov/ncidod/dhep/gl_ isolation.html

5. Centers for Disease Control and Prevention. Division of healthcare Quality promotion. Surveillance of healthcare personnel with HIV/AIDS [serial online] 2005. Available from: http:// www.cdc.gov/ncidod/hip/Blood/hivpersonnel.htm
E, na análise da regressão logística multivariada, não foi possível identificar nenhuma variável estatisticamente associada a ocorrência de acidente com exposição a material biológico $(\mathrm{p}<0,05$; IC95\%). Observou-se também Que para a variável estar lotado em unidade de suporte avançado de vida manteve associação estatisticamente significante, porém com um índice de confiabilidade de 90\% ( $<<0$, I; IC 90\%). Em estudo similar realizado em Belo Horizonte registrou-se uma associação para esta variável de $95 \%$, sendo possível inferir Que estar lotado neste tipo de unidade pode aumentar o risco da ocorrência de acidentes de acidentes para o trabalhador do APH.

\section{CONCLUSÃO}

Pelo presente estudo verificou-se Que a incidência global de acidentes envolvendo material biológico foi de 19,8\%. Dentre os profissionais acidentados, $56,5 \%$ acidentaram por contato com fluidos corporais, apenas 30,4\% realizaram avaliação médica pósacidente, $8,7 \%$ dos casos a emissão da CAT foi realizada, em 52,2\% nenhuma conduta foi tomada imediatamente; e também, na mesma proporção dos acidentados, o acompanhamento sorológico recomendado não foi realizado. A análise univariada revelou Que profissionais com idade superior a 31 anos e aqueles lotados em Unidades de Suporte Avançado foram as variáveis Que apresentaram associação estatisticamente significante a ocorrência de acidentes de trabalho.

Sugere-se a realização de programas de educação permanente, visando minimizar a ocorrência do acidente e reforçar Quanto a importância de sua notificação através da emissão da CAT. Considera-se Que, paralelo a essa iniciativa, a instituição juntamente com os profissionais Que atuam nesta área possam construir e implantar um protocolo formal de orientação e acompanhamento dos profissionais acidentados por exposição a material biológico.
6. Ministério da Saúde (BR). Portaria $\mathrm{n}^{\circ}$. 2048 de 5 de setembro de 2002. Dispõe sobre a organização do atendimento móvel de urgência - SAMU. Diário Oficial da União 2002 set 6.

7. Florêncio VB, Rodrigues CA, Pereira MS, Souza ACS. Adesão as precauções padrão entre os Profissionais da Equipe de Resgate Pré-Hospitalar do Corpo de Bombeiros de Goiás. Rev Eletrônica Enferm 2003; 5(1). [serial online]. Disponível em: http://www.fen.ufg.br/revista/revista5_l/adesao.html

8. Paiva, MHRS. Atendimento pré-hospitalar público de Belo Horizonte: uma análise da adoção às medidas de precaução pela equipe multidisciplinar [dissertação]. Belo Horizonte: Escola de Enfermagem, Universidade Federal de Minas Gerais; 2007.

9. Zapparoli AS, Marziale MHP. Risco ocupacional em unidades de Suporte Básico e Avançado de Vida em Emergências. Rev Bras Enferm 2006; 59(1): 4I-6.

10. Sunmi P, Ihnsook I, Jeongae H, Yunkyoung Y, Sunhee L, Changhwa C. Needlestick and sharps injuries in a tertiary hospital in the Republic of Korea. Am I Infect Control 2008; 36(6): 439-43.

11. Shimizu HE, Ribeiro EJG. Ocorrência de acidente de trabalho por materiais perfurocortantes e fluídos biológicos em estudantes e trabalhadores da saúde de um hospital escola de Brasília. Rev 
Esc Enferm USP 2002; 36(4): 367-75.

12. Sarquis LMM. Monitoramento de trabalhadores de saúde expostos aos fluidos biológicos [tese]. São Paulo: Escola de Enfermagem, Universidade de São Paulo; 2007.

13. Baets AJ, Sifovo S, Pazvakavambwa IE. Access to occupational postexposure prophylaxis for primary health care workers in rural Africa: a cross-sectional study. Am I Infect Control 2007; 35(8): 545-51.

14. Damasceno AP, Pereira MS, Souza ACS, Tipple AFV, Prado MA. Acidentes ocupacionais com material biológico: a percepção do profissional acidentado. Rev Bras Enferm 2006; 59(1): 72-7.

15. Takeda E, Robazzi MLCC. Acidentes de trabalho com motoris- tas de ambulâncias Que realizam socorro de urgência. Rev Latino-am Enfermagem 2007; 15(3).

16. Nhamba LA. Acidentes ocupacionais com material biológico entre profissionais de enfermagem em um hospital em Angola [dissertação]. Ribeirão Preto: Escola de Enfermagem de Ribeirão Preto, Universidade de São Paulo; 2004.

17. Nishide VM, Benatti MAC, Alexandre NMC. Ocorrência de acidente do trabalho em uma unidade de terapia intensiva. Rev Latino-am Enfermagem 2004; 12(2): 204-I I.

18. Mathews R, Leiss IK, Lyden IT, Sousa S, Ratcliffe JM, Jagger J. Provision and use of personal protective equipment and safety devices in the National Study to Prevent Blood Exposure in Paramedics. Am I Infect Control 2008; 36( I0): 743-9. 